\title{
Prior Adjuvant Endocrine Therapy
}

National Cancer Institute

\section{Source}

National Cancer Institute. Prior Adjuvant Endocrine Therapy. NCI Thesaurus. Code C15565.

An indication that an individual has been previously treated with endocrine/hormonal therapy following surgery. 